\title{
Reabilitação cardiopulmonar e metabólica: aspectos práticos e responsabilidades
}

\author{
Editor: Tales de Carvalho \\ Colaboradores: Alberto Aguillar Cortez, Almir Ferraz, Antonio Claudio Lucas da Nóbrega, \\ Antonio Fernando Brunetto, Artur Haddad Herdy, Carlos Alberto Cordeiro Hossri, Carlos Alberto Neder, \\ Carlos Eduardo Negrão, Cláudio Gil Soares Araújo, Fábio Sândoli de Brito, Félix Albuquerque Drummond, \\ Fernando Roselino, Geraldo Ângelo Nogueira, Iracema Ioco Kikuchi Umeda, J apy Angelini Oliveira Filho, \\ J osé Antonio Caldas Teixeira, J osé Kawazoe Lazzoli, Luiz Eduardo Mastrocolla, Magnus Benetti, \\ Marcelo Bichels Leitão, Marcelo Zager, Maria J anieire Nazaré Nunes Alves, Max Grinberg, Miltom Godoy, \\ Odwaldo Barbosa e Silva, Ricardo Stein, Ricardo Vivacqua Costa, Romeu Sergio Meneghelo, \\ Ruy Silveira Moraes, Salvador Manoel Serra e Salvador Ramos
}

\section{INTRODUÇÃO}

Este documento completa a trilogia de normatizações da Sociedade Brasileira de Cardiologia sobre reabilitação cardiovascular, juntando-se à Nomatização dos Equipamentos e Técnicas da Reabilitação Cardiovascular Supervisionada, de 2004(1), e à Diretriz de Reabilitação Cardíaca, de 2005(2). Foi elaborado em sintonia com o Projeto de Reabilitação Cardiovascular, Pulmonar e Metabólica (RCPM), fruto da parceria do Departamento de Ergometria e Reabilitação Cardiovascular da Sociedade Brasileira de Cardiologia com o SBC-Funcor, desenvolvido com o apoio da Sociedade Brasileira de Medicina do Esporte, tendo como objetivos: a) proporcionar treinamento em RCPM; b) implantar programas estruturados de RCPM no sistema de saúde público e privado; c) desenvolver estudos científicos multicêntricos sobre RCPM. É um documento produzido com a explícita intenção de contribuir para o surgimento de ações políticas nas quais a RCPM seja priorizada, algo imprescindível para a saúde pública, sendo relevante também em termos econômicos ${ }^{(3,4)}$. Não tem dentre seus objetivos abordar aspectos específicos da avaliação física e da prescrição de exercício, considerando que tais informações estão devidamente contempladas na I Diretriz Nacional de Reabilitação, de $1997^{(5)}$, e em outros documentos da Sociedade Brasileira de Cardiologia( ${ }^{(1,2)}$.

O enfoque abrangente justifica a denominação RCPM e está em consonância com a Organização Mundial de Saúde, que caracteriza a reabilitação como a integração de intervenções, denominadas "ações não farmacológicas", para assegurar as melhores condições físicas, psicológicas e sociais para o paciente com doença cardiovascular, pulmonar e metabólica(3,4). A prática médica ética, com base em evidências científicas e de comprovadas vantagens de ordem econômica, não admite deixar sem o benefício terapêutico da RCPM os portadores de doenças cardiovasculares, como a doença coronária aterosclerótica(6-12), insuficiência cardíaca(13-15), hipertensão arterial sistêmica ${ }^{(16)}$ e doença arterial periférica(17-19); doenças metabólicas ${ }^{(20-28)}$, como a obesidade, síndrome metabólica e diabete melito; doença pulmonar crônica(29-33); de fatores de risco relevantes para as referidas doenças, como o tabagismo, dislipidemia, intolerância à glicose, excesso de estresse, sedentarismo de longa data(20,34-37); e de nefropatia crônica(38-42). Neste contexto, destaca-se a necessidade da prescrição de exercício físico, que em determinadas situações deve ocorrer sob supervisão especializada(43-45).

Apesar da RCPM ser considerada modalidade terapêutica segura ${ }^{(43)}$, obrigatória pelo que significa em temos de custo-efetividade $^{(8-10,12,14,28,31-33,37,39)}$, no Brasil, refletindo desinformação e/ou má atitude política, tanto no setor público quanto privado, os benefícios dos programas estruturados de reabilitação são ainda pouco mobilizados em prol dos pacientes. Freqüentemente os planos de saúde costumam tratar o processo de reabilitação com as mes- mas regras estabelecidas para procedimentos fisioterápicos, destinados a tratar problemas músculo-esqueléticos agudos, por vezes permitindo ao paciente apenas 10 sessões por ano, desconsiderando que devido à sua complexidade a RCPM deve ser regida segundo normas próprias, que estão bem estabelecidas pela comunidade médica e científica, nacional e internacional(1-5).

$\checkmark$ No Brasil, impõe-se o surgimento de um novo paradigma cultural e político, que priorize a RCPM , o que beneficiaria a saúde orgânica dos pacientes e a saúde econômica do sistema de saúde.

\section{GRAU DE RECOMENDAÇÃO, NÍVEL DE EVIDÊNCIA, ASPECTOS ECONÔMICOS}

Estudos científicos, conclusivos sobre a redução significativa da morbimortalidade e melhoria da qualidade de vida dos pacientes tratados, têm associado RCPM e custo-efetividade(8-10,12,14,28,31$33,37,46)$. Na reabilitação de portadores de cardiopatias existem duas estratégias básicas: a que prioriza o exercício, reconhecida na literatura internacional pela sigla EX-CR, de "exercise-only", que pode ser traduzida para reabilitação cardíaca com ênfase no exercício (RCEE); e a que é reconhecida pela sigla CCR, de "comprehensive care rehabilitation", que pode ser traduzida para reabilitação cardíaca abrangente (RCA), na qual o exercício é parte de um conjunto que, juntamente com o condicionamento físico, objetiva a remoção do tabagismo, a reformulação de hábitos alimentares e o controle do estresse. Independente da estratégia, a RCPM estruturada corresponde a processo educativo, em contexto no qual o paciente deve ser provido de informações básicas sobre a fisiopatologia de sua(s) doença(s); relação da(s) doença(s) com atividade física, atividade sexual e trabalho; mecanismos de ação dos fármacos; reformulação dos hábitos alimentares; cessação do tabagismo e controle do estresse. Cabe enfatizar que as evidências científicas dão relevância ao treinamento físico, credenciando-o como a principal intervenção neste processo de reabilitação(8).

No Brasil, o custo da intervenção coronária percutânea (ICP) com colocação de apenas um STENT com eluição de medicamento é de aproximadamente $\mathrm{R} \$ 16.000,00$. Com este recurso, caso a reabilitação custasse $R \$ 300,00$ mensais, seriam possíveis mais de quatro anos de programa supervisionado para um paciente e o atendimento de mais do que 50 pacientes/mês em programa estruturado de RCPM. Freqüentemente na ICP utiliza-se mais do que um STENT, tornando ainda mais díspares os custos das duas modalidades terapêuticas. Deve ser ressaltado que os benefícios advindos da reabilitação são seguros e abrangentes, melhorando qualidade de vida e proporcionando maior redução das mortalidades cardíaca e total, em estudos que a comparam com a ICP, conforme pode ser observado na tabela $1^{(8,11)}$. 
TABELA 1

Custo-efetividade de intervenções terapêuticas em cardiologia

\begin{tabular}{|c|c|c|}
\hline Intervenção & Fator de comparação & Custo-efetividade \\
\hline Programa antitabagismo & Sem de terapia & US\$ 220 por VSA \\
\hline Programas de educação para a redução do colesterol & Sem intervenção & US\$3.475 por VSA \\
\hline $\begin{array}{l}\text { Reabilitação Cardíaca } \\
\text { (Incluso estudo de Ades et al., 1997) }\end{array}$ & Cuidado habitual & US\$ 4.950 por VSA \\
\hline ICP (uma artéria, angina severa) & Cuidados médicos & US\$ 8.700 por GQVA \\
\hline Redução lipídica (sinvastatina) para prevenção secundária & Sem terapia & US\$9.630 por VSA \\
\hline $\begin{array}{l}\text { Reabilitação Cardíaca } \\
\text { (incluso estudo de Lowenstyn et al., 2000) }\end{array}$ & Cuidados rotineiros & US\$ 15.000 por VSA \\
\hline $\begin{array}{l}\text { Reabilitação Cardíaca } \\
\text { (incluso estudo de Oldridge, 1993) }\end{array}$ & Cuidados usuais & $\begin{array}{l}\text { US } \$ 21.800 \text { por VSA } \\
\text { US\$ } 9.200 \text { por GQVA }\end{array}$ \\
\hline Cirurgia de RM & ICP & US\$26.570 por VSA \\
\hline Ativador de plasminogênio tecidual (t-PA) & Estreptoquinase & US\$35.275 por VSA \\
\hline Reperfusão trombolítica (t-PA, IAM anterior, idade 41-60) & Estreptoquinase & US\$ 49.900 por VSA \\
\hline Captopril (para pacientes com mais de 50 anos sobreviventes de IAM ) & Sem captopril & US\$ 76.000 por GQVA \\
\hline ICP (uma artéria, angina leve) & Cuidados médicos & US\$126.400 por GQVA \\
\hline \multicolumn{3}{|c|}{$\begin{array}{l}\text { VSA - Vida salva por ano } \\
\text { GQVA - Ganho de qualidade de vida por ano } \\
\text { RM - Revascularização miocárdica } \\
\text { ICP - Intervenção coronária percutânea } \\
\text { IAM - Infarto agudo do miocárdio } \\
\text { Ades PA, Pashkow FJ, Nestor J R. Cost-effectiveness of cardiac rehabilitation after myocardial infarction. J Cardiopulm Rehabil 1997;17:222-31. } \\
\text { Chapman RH, Stone PW, Sandberg EA, Bell C, Neumann PJ . A comprehensive league table of cost-utility ratios and a sub-table of "panel-worthy" studies. } \\
\text { Med Decis Making 2000;20:451-67. } \\
\text { O'Brien BJ . Chapter 3. Cost effectiveness of ICD therapy: a review of published evidence. Can J Cardiol 2000;16:1307-12. }\end{array}$} \\
\hline
\end{tabular}

Os bons resultados do tratamento por meio da RCPM aplicado a portadores das doenças referidas neste documento têm sido documentados por estudos consistentes e de boa qualidade, com a evidência científica variando entre os níveis 1 e 3 , segundo classificação proposta pela Comissão de Medicina Baseada em Evidência, da Sociedade Brasileira de Cardiologia (SBC) e Associação Médica Brasileira (AMB) (quadro 1). Considerando a qualidade das evidências científicas e a aplicabilidade do método ${ }^{(3,4)}$, o tratamento por meio da RCPM para estes pacientes apresenta sempre forte grau de recomendação (grau A ou grau B), segundo classificação proposta pela comissão da SBC e AMB citada (quadro 2).

\section{QUADRO 1}

Nível de evidência

Nível 1: evidência baseada em muitos estudos randomizados, controlados, amplos, concordantes e com poder estatístico adequado; preferencialmente com revisão sistemática conclusiva.

Nível 2: evidência baseada em poucos estudos randomizados, controlados, concordantes e de médio porte ou metanálises de vários estudos desta natureza, pequenos ou de médio porte.

Nível 3: evidência baseada em poucos estudos randomizados, controlados e de ótima qualidade.

Nível 4: evidência baseada em mais de um estudo coorte, de ótima qualidade.

Nível 5: evidência baseada em mais de um estudo caso-controle, de qualidade.

Nível 6: evidência baseada em mais de uma série de casos de alta qualidade. Inclui registros.

Nível 7: evidência baseada apenas em: extrapolações de resultados coletados para outros propósitos (testar outras hipóteses); conjecturas racionais, experimentos com animais, ou baseados em modelos mecanísticos de fisiopatologia e/ou mecanismos de ação; conduta antiga baseada em prática comum; opiniões sem referência a estudos anteriores.

Fonte: Comissão de Cardiologia Baseada em Evidência da SBC e AMB.

\section{QUADRO 2}

Grau de recomendação

A =Sempre usar. Recomendação conclusiva, sendo adotada por unanimidade; conduta conclusivamente útil e segura; eficácia e segurança comprovadas. Quase sempre se requer níveis de evidência 1 ou 2 para que este grau de recomendação seja adotado.

B = Deve ser geralmente indicada. Recomendação considerada aceitável, mas com ressalvas; conduta aceitável e segura; grande potencial de utilidade, mas ainda sem comprovação conclusiva, com nível de evidência menos sólido.

C = Fica a critério pessoal usar. Recomendação indefinida; conduta a respeito da qual não há evidência segura a favor ou contra, quanto à eficácia e segurança.

D = Em geral não se deve usar. Conduta não recomendada, embora possa em algum contexto excepcional ser adotada, tratando-se de opção muito fraca; evidência mínima de eficácia e segurança, embora se vislumbre algum potencial de utilidade em algumas circunstâncias.

$\mathbf{E}=$ Nunca usar. Não recomendada por unanimidade.

Fonte: Comissão de Cardiologia Baseada em Evidência da SBC e AMB.

\section{$\checkmark$ RCPM na coronariopatia: recomendação grau A, evidên- cia de nível 1}

A indicação da reabilitação para coronariopatas torna-se inquestionável diante das evidências dos estudos sobre custo-efetividade ${ }^{(8-10)}$ e do número de indivíduos que necessitam ser tratados para que o benefício do tratamento (morte e/ou infarto do miocárdio não fatal evitados) ocorra em um paciente(9-10,47-51), o que corresponde à sigla NNT, de "number needed to treat" (quadros 3 e 4).

\section{$\checkmark$ RCPM na insuficiência cardíaca: recomendação grau A, evidência nível 1}

Em pacientes com insuficiência cardíaca os estudos sobre o custo-efetividade do tratamento por meio da RCPM têm mostrado resultados mais expressivos do que os referentes a coronario- 
patas $^{(12-14)}$. Nos pacientes mais graves, com indicação para transplante cardíaco, a reabilitação prévia à cirurgia pode melhorar as condições gerais, tornando mais seguros o ato cirúrgico e o pósoperatório. Nestes casos o objetivo maior volta-se à melhora da capacidade funcional, que auxilia na otimização do tratamento e por vezes posterga ou torna desnecessário o transplante cardíaco.

\section{QUADRO 3}

NNT de alguns dos principais recursos medicamentosos e da reabilitação cardíaca aplicados a indivíduos pós-infarto agudo do miocárdio

$\checkmark$ Reabilitação cardíaca: NNT - 66 e 49 (95\% Cl: 26 to 120)(1)

$\checkmark$ Betabloqueador após IAM: NNT $=84^{(2)}$

$\checkmark$ Antiadesivo plaquetário após IAM : NNT $=306^{(3)}$

$\checkmark$ Estatina: NNT $=11$ a $56^{(4-7)}$

1. Oldridge N, et al. Number needed to treat in cardiac rehabilitation. J Cardiopulm Rehabil 2002; 22:22-30.

2. Freemantle $\mathrm{N}$, et al. Beta blockade after myocardial infarction: systematic review and meta regression analysis. BMJ 1999;318:1730-7.

3. Antiplatelet Trialists' Collaboration. Collaborative overview of randomized trials of antiplatelet therapy - Prevention of death, myocardial infarction, and stroke by prolonged antiplatelet therapy in various categories of patients. BMJ 1994;308:81-106.

4. Randomized trial of cholesterol lowering in $\mathbf{4 4 4 4}$ patients with coronary heart disease: the Scandinavian Sinvastatin Survival Study (4S). Lancet 1994;344:1383-9.

5. Kumana CR, et al. Gauging the impact of statins using number needed to treat. J AMA 1999; 282:1899-901

6. Long-term Intervention with Pravastatin in Ischaemic Disease (LIPID) Study Group. Prevention of cardiovascular events and death with pravastatin in patients with coronary heart disease and a broad range of initial cholesterol levels. N Engl J Med 1998;339:1349-57.

7. Wilson TM, Tanaka H. Meta-analysis of the age-associated decline in maximal aerobic capacity in men: relation to training status. Am J Physiol Heart Circ Physiol 2000;278:H829-34.

* NNT, de " number needed to treat", que corresponde ao número de indivíduos que necessitam ser tratados para que o benefício do tratamento, no caso morte evitada, ocorra em um paciente.

** Os estudos com antiadesivo plaquetário e betabloqueador corresponderam a seguimentos de apenas 1 ano, enquanto os estudos com estatina e reabilitação a seguimento por período maior

\section{QUADRO 4}

NNT* anual** para reduzir mortalidade total por meio da reabilitação cardíaca e de alguns dos principais recursos medicamentosos indicados no tratamento pós-infarto do miocárdio

> Reabilitação cardíaca: NNT = 112-187

Oldridge N, et al. Number needed to treat in cardiac rehabilitation. J Cardiopulm Rehabil 2002; 22:22-30.

> Betabloqueadores: NNT $=84$

Freemantle $\mathrm{N}$, et al. Beta blockade after myocardial infarction: systematic review and meta regression analysis. BMJ 1999;318:1730-7.

$>$ Antiadesivo plaquetário: NNT $=306$

Antiplatelet Trialists' Collaboration. Collaborative overview of randomized trials of antiplatelet therapy - Prevention of death, myocardial infarction, and stroke by prolonged antiplatelet therapy in various categories of patients. BMJ 1994;308:81-106.

$>$ Estatina (sinvastatina): NNT $=164$

Randomized trial of cholesterol lowering in 4444 patients with coronary heart disease: the Scandinavian Simvastatin Survival Study (4S). Lancet 1994;344:1383-9.

> Estatina (pravastatina): NNT $=197$

Long-term Intervention with Pravastatin in Ischaemic Disease (LIPID) Study Group. Prevention of cardiovascular events and death with pravastatin in patients with coronary heart disease and a broad range of initial cholesterol levels. N Engl J Med 1998;339:1349-57.

* NNT, de " number needed to treat", que corresponde ao número de indivíduos que necessitam ser tratados para que o benefício do tratamento, no caso morte evitada, ocorra em um paciente.

** Os dados de todos os estudos estão ajustados para seguimento de 1 ano, permitindo melhor comparação entre os distintos tratamentos.

\section{$\checkmark$ RCPM na pneumopatia crônica: recomendação grau A, evidência de nível 1}

Pneumopatas crônicos tratados em programas de reabilitação pulmonar utilizam menos os serviços de saúde. Os custos de implantação e demais gastos referentes aos programas de reabilitação são vantajosamente compensados, gerando bom retorno eco- nômico ao sistema de saúde. A conciliação da vantagem econômica com o incremento da quantidade e da qualidade de vida, como decorrência de comprovados ganhos na capacidade funcional e estado geral dos pneumopatas crônicos, torna obrigatória a indicação complementar de tratamento em programa de reabilitação(18-21).

\section{$\checkmark$ RCPM na hipertensão arterial sistêmica: recomendação de grau $A$, evidência de nível 1}

Estudos controlados e randomizados reunidos em uma grande metanálise ${ }^{(16)}$ documentaram a redução significativa da pressão arterial sistólica e diastólica, não somente em portadores de hipertensão arterial sistêmica, mas também em indivíduos nomotensos.

\section{$\checkmark$ RCPM na doença arterial obstrutiva periférica, obesida- de, síndrome metabólica, diabete melito e para pacientes com escore elevado de risco para doença cardiovascular, pulmonar e metabólica: recomendação de grau A-B, evidên- cia de nível 2-3}

Os estudos científicos têm sido conclusivos em relação aos benefícios da RCPM para indivíduos com doença arterial obstrutiva periférica, obesidade, diabete melito, síndrome metabólica e risco elevado para as doenças cardiovasculares, pulmonares e metabólicas (19,20-28,34-37,39). Para o estabelecimento do grau de risco tem sido recomendada avaliação sistemática, que pode ser aplicada por meio de escores como os de Framingham e da Sociedade Européia de Cardiologia, dentre outros ${ }^{(52)}$.

\section{$\checkmark$ RCPM na nefropatia crônica, inclusive para pacientes sub- metidos a programa de diálise: recomendação de grau B, evidência de nível 3}

Tem sido também demonstrada a importância da reabilitação para nefropatas crônicos, inclusive os submetidos a programa de hemodiálise, os quais apresentam acentuada redução da capacidade cardiorrespiratória(38). Para esses, a prática de exercícios físicos proporciona a redução da pressão arterial sistólica, aumento da capacidade física e redução do custo do tratamento(39). Os pacientes com insuficiência renal costumam desenvolver doença cardiovascular, independente da modalidade de tratamento escoIhida, seja hemodiálise ou transplante renal, sendo que o exercício físico contribui para reduzir ou retardar problemas secundários à coronariopatia, hipertensão arterial sistêmica e insuficiência cardíaca ${ }^{(40)}$. Em pacientes submetidos ao tratamento dialítico, o exercício proporciona melhora da função cardiovascular, da capacidade física e da qualidade de vida, melhorando a eficiência da diálise e diminuindo o tempo de remoção de fosfato durante a diálise ${ }^{(41)}$, reduzindo a mortalidade ${ }^{(42)}$.

\section{IMPLANTAÇÃO DE PROGRAMAS. ASPECTOS PRÁTICOS}

A comprovada repercussão clínica e econômica da RCPM obriga a implementação de políticas no sistema de saúde público e privado, que tornem o método disponível a todos os pacientes que preencherem os critérios de indicação.

Neste contexto, é essencial que todos os hospitais com serviço de cardiologia possuam programa estruturado de RCPM, para início do processo enquanto o paciente ainda estiver internado (fase 1 da reabilitação).

Torna-se imperioso que os centros de saúde da rede pública disponham de locais próprios para a orientação do exercício físico, com estrutura e recursos humanos capacitados para o atendimento de pacientes elegíveis para as fases extra-hospitalares da RCPM permitindo: a) o desenvolvimento da fase 2 , de duração mínima de três meses, imediatamente após a alta, objetivando incrementar a capacidade física, por meio de sessões supervisionadas; b) desenvolvimento das fases 3 e 4, sendo a fase 3 um período ain- 
da de franco aprimoramento físico, com duração prevista entre seis meses e um ano e a fase 4 um período principalmente de manutenção dos ganhos obtidos e de diversificação do repertório, de duração muito variável. A equipe básica deve ser composta por profissionais com treinamento em RCPM, devendo ser composta pelo menos por um médico (responsável, coordenador), um professor de educação física e/ou fisioterapeuta e um profissional da área de enfermagem. Os referidos profissionais não necessitariam dedicar tempo integral ao programa de reabilitação, que poderia funcionar, por exemplo, três a cinco vezes por semana, duas horas cada dia, podendo exercer outras funções no sistema de saúde. São desejáveis ações que integrem o programa de reabilitação com as demais atividades do centro de saúde, como o programa de saúde da família. Sempre que houver possibilidade, a equipe deve contar, também, com a participação de terapeuta ocupacional e assistente social, os quais, juntamente com os profissionais já citados, comporiam a equipe de RCPM ideal.

Na capacitação dos recursos humanos, deve ser considerada a necessidade de conhecimentos básicos sobre fisiologia do exercício, que permitam a interpretação das modificações fisiológicas decorrentes do treinamento. É importante que os membros da equipe consigam, previamente ao início, e no curso das sessões de exercício, identificar sintomas e sinais de alerta, correspondentes a situações que impliquem risco para os pacientes, como, por exemplo, hiper e hipoglicemias, broncoespasmo, queda da saturação de oxigênio, episódios de isquemia miocárdica, arritmias cardíacas graves, hipertensão arterial descontrolada, etc. Para todos os profissionais exige-se formação que permita o adequado atendimento de emergências, o que inclui treinamento básico em reanimação cardiorrespiratória.

\section{TABELA 2}

Doenças elegíveis para Reabilitação Cardiopulmonar e Metabólica com os códigos da CBHPM que servem para reabilitação

\begin{tabular}{|c|c|c|c|}
\hline Código & Descrição do procedimento & Porte & $\begin{array}{l}\text { Custo } \\
\text { oper. }\end{array}$ \\
\hline 2.01.03.22-0 & Doenças pulmonares atendidas em ambulatório & $1 \mathrm{~B}$ & 0,40 \\
\hline 2.01.03.24-7 & Exercícios para reabilitação do asmático & $1 \mathrm{~A}$ & 0,440 \\
\hline $2.01 .03 .35-2^{\#}$ & $\begin{array}{l}\text { Paciente em pós-operatório de cirurgia cardíaca, } \\
\text { atendido em ambulatório }\end{array}$ & $1 \mathrm{~B}$ & 1,060 \\
\hline $2.01 .03 .36-0^{\#}$ & $\begin{array}{l}\text { Paciente com DPOC em atendimento } \\
\text { ambulatorial necessitando de reeducação e } \\
\text { reabilitação respiratória }\end{array}$ & $1 \mathrm{C}$ & 0,540 \\
\hline $2.01 .03 .37-9^{\#}$ & $\begin{array}{l}\text { Paciente em pós-operatório de cirurgia cardíaca, } \\
\text { atendido em ambulatório }\end{array}$ & $1 \mathrm{~B}$ & 1,060 \\
\hline $2.01 .03 .38-7$ & $\begin{array}{l}\text { Paciente com doença isquêmica do coração } \\
\text { atendido em ambulatório }\end{array}$ & $1 \mathrm{~B}$ & 1,060 \\
\hline $2.01 .03 .39-5$ & $\begin{array}{l}\text { Paciente com doença isquêmica do coração } \\
\text { atendido em ambulatório }\end{array}$ & $1 \mathrm{~B}$ & 1,060 \\
\hline $2.01 .03 .41-7$ & $\begin{array}{l}\text { Paciente sem doença coronariana clinicamente } \\
\text { manifesta, mas considerado de alto risco } \\
\text { atendido em ambulatório }\end{array}$ & $1 \mathrm{C}$ & 1,060 \\
\hline $2.01 .03 .58-1$ & $\begin{array}{l}\text { Programa de exercícios supervisionados com } \\
\text { obtenção de ECG e/ou saturação de oxigênio }\end{array}$ & $1 \mathrm{~A}$ & 1,060 \\
\hline $2.01 .03 .60-3$ & $\begin{array}{l}\text { Programa de exercícios supervisionados sem } \\
\text { obtenção de ECG e/ou saturação de oxigênio }\end{array}$ & & \\
\hline $2.01 .03 .72-7^{\#}$ & Reabilitação cardíaca supervisionada. & $1 \mathrm{C}$ & 1,060 \\
\hline $2.01 .03 .06-3$ & $\begin{array}{l}\text { Paciente com doença isquêmica do coração } \\
\text { hospitalizado }\end{array}$ & $1 \mathrm{~B}$ & 1,060 \\
\hline $2.02 .03 .07-1$ & $\begin{array}{l}\text { Paciente em pós-operatório de cirurgia cardíaca, } \\
\text { hospitalizado }\end{array}$ & $1 \mathrm{~B}$ & 1,060 \\
\hline $2.01 .03 .21-2$ & $\begin{array}{l}\text { Distúrbios circulatórios arteriais (ex. doença } \\
\text { arterial periférica), venosas (ex. varizes) } \\
\text { e linfáticas }\end{array}$ & $1 \mathrm{~B}$ & 0,440 \\
\hline
\end{tabular}

\# códigos mais usados.

Fonte: Adaptado de CBHPM 3a edição.

Observação: foram retirados os tempos estabelecidos para alguns dos procedimentos, sendo considerados os prazos estabelecidos neste documento, como o tempo previsto para as diversas fases da RCPM.
Para que os pacientes da rede privada tenham o atendimento básico necessário visando às prevenções primária e secundária das doenças cardiovasculares, pulmonares e metabólicas, os planos privados de saúde devem contemplar a RCPM em suas quatro fases, cujas características serão mais bem descritas adiante. Deve servir como base para as cobranças o que consta na tabela 2.

\section{Fases da reabilitação}

A RCPM faz parte do esquema geral de tratamento médico, devendo sempre ser considerada dentro deste contexto. Portanto, para o seu início impõe-se o encaminhamento e consentimento do médico assistente. Cabe ao coordenador/responsável médico a liberação do paciente para iniciar as atividades e a alta de cada fase da reabilitação, sempre em sintonia com o médico assistente do paciente.

FASE 1 - Aplica-se ao paciente internado. É o passo inicial em direção a uma vida ativa e produtiva. Nos primórdios foi destinada à recuperação após infarto do miocárdio ou cirurgia de revascularização miocárdica (RM). Atualmente deve incluir os pacientes submetidos às intervenções coronárias percutâneas (ICP) por técnica de balão ou implante de STENT, cirurgias para valvopatia, cirurgias para cardiopatia congênita, transplante cardíaco, paciente com angina do peito de caráter estável e paciente com fatores de risco para doença coronária. Esta fase destina-se também aos diabéticos, hipertensos, portadores de síndrome metabólica, nefropatas crônicos e pneumopatas crônicos, internados devido a descompensação clínica. Interessa, portanto, aos pacientes internados por descompensação clínica de natureza cardiovascular, pulmonar e metabólica. A Fase 1 inicia-se após o paciente ter sido considerado compensado clinicamente, como decorrência da otimização do tratamento clínico e/ou utilização de procedimento intervencionista. Devem predominar a combinação de exercício físico de baixa intensidade, técnicas para o controle do estresse e programas de educação em relação aos fatores de risco. A duração desta fase tem decrescido nos anos recentes, em decorrência de internações hospitalares mais curtas. É ideal que a equipe de profissionais seja composta por médico, fisioterapeuta, enfermeiro, nutricionista e psicólogo. A equipe básica deve ser composta pelo menos por um médico (responsável, coordenador), um fisioterapeuta e um profissional da área de enfermagem. Todos os profissionais devem ter sido submetidos a treinamento em RCPM. Os profissionais não necessitam dedicar tempo integral ao programa de reabilitação, podendo exercer outras atividades no hospital. 0 programa nesta fase objetiva que o paciente tenha alta hospitalar com as melhores condições físicas e psicológicas possíveis, municiado de informações referentes ao estilo saudável de vida, em especial no que diz respeito ao processo de RCPM.

FASE 2 - É a primeira etapa extra-hospitalar. Inicia-se imediatamente após a alta e/ou alguns dias após um evento cardiovascular ou descompensação clínica de natureza cardiovascular, pulmonar e metabólica. Duração prevista: três a seis meses, podendo em algumas situações se estender por mais tempo. Pode funcionar em estrutura que faça parte do complexo hospitalar ou outro ambiente próprio para a prática de exercícios físicos (clube esportivo, ginásio de esportes, sala de ginástica, etc.). A equipe ideal deve incluir médico, fisioterapeuta, professor de educação física, enfermeiro, nutricionista e psicólogo. Deve contar com os recursos básicos para o atendimento de emergências. Funciona com sessões supervisionadas pelo fisioterapeuta e/ou professor de educação física. O programa de exercícios deve ser individualizado, em termos de intensidade, duração, freqüência, modalidade de treinamento e progressão. Sempre devem existir recursos para a correta determinação da freqüência cardíaca e verificação de pressão arterial, além da possibilidade de eventual verificação da saturação de oxigênio, determinação da glicemia e monitoração eletrocardiográfica. Fazem parte desta fase um programa educacional 
direcionado à modificação do estilo de vida, com ênfase na reeducação alimentar e estratégias para cessação do tabagismo. A reabilitação nesta fase tem como principal objetivo contribuir para o mais breve retomo do paciente às suas atividades sociais e laborais, nas melhores condições físicas e emocionais possíveis.

FASE 3 - Duração prevista: seis a 24 meses. Destina-se a atender imediatamente os pacientes liberados da fase 2 , mas pode ser iniciada em qualquer etapa da evolução da doença, não sendo obrigatoriamente seqüência das fases anteriores. Portanto, pacientes de baixo risco que não participaram da fase 2 são bons candidatos. A supervisão de exercícios deve ser feita por profissional especializado em exercício físico (professor de educação física e/ ou fisioterapeuta). Deve sempre contar com a coordenação geral de um médico e dispor de condições para eventual monitoração cardíaca e determinação da saturação de oxigênio. É recomendável que faça parte da equipe profissional de enfermagem e, caso haja disponibilidade, a equipe pode ser completada por nutricionista e psicólogo. O principal objetivo é o aprimoramento da condição física, mas deve ser considerada também a necessidade de promoção de bem-estar (melhora da qualidade de vida) e demais procedimentos que contribuam para a redução do risco de complicações clínicas, como é o caso das estratégias para cessação do tabagismo e reeducação alimentar.

FASE 4 - É um programa de longo prazo, sendo de duração indefinida, muito variável. As atividades não são necessariamente supervisionadas, devendo ser adequadas à disponibilidade de tempo para a manutenção do programa de exercícios físicos e às preferências dos pacientes em relação às atividades desportivas recreativas. Devem ser igualmente considerados os recursos materiais e humanos disponíveis. Nesta fase os pacientes, após cada avaliação médica, principalmente quando são submetidos a testes ergométricos, cuja periodicidade não deve exceder a um ano, devem ser avaliados e orientados na prática, sempre que possível com algumas sessões supervisionadas de exercícios. Os objetivos principais desta fase são o aumento e a manutenção da aptidão física. Não há obrigatoriedade de que esta fase seja precedida pela fase 3. A equipe da reabilitação deve propor a programação de atividades que seja mais apropriada, prescrevendo a carga de exercícios que atenda às necessidades individuais. Os pacientes devem ser periódica e sistematicamente contatados pela equipe do programa de RCPM, mesmo que por telefone, pelo menos uma vez a cada seis meses. Deve ser considerada a possibilidade de atividades em grupo, aproveitando, por exemplo, o calendário de atividades educacionais dirigidas à população.

REABILITAÇÃO NÃO SUPERVISIONADA - Tendo em vista a escassez de centros estruturados de reabilitação entre nós, o que gera grande demanda reprimida de pacientes crônicos estáveis, deve ser considerada a possibilidade de que a RCPM seja aplicada por meio de programa de reabilitação não supervisionada (RCPMNS), que poderia ser chamada de reabilitação parcialmente supervisionada (RCPM-PS), o que talvez corresponda mais à realidade. Enfatizamos que a adoção da RCPM-NS mantém a necessidade da prescrição individualizada de exercícios, que deve ser acompanhada de demonstrações práticas, em sessões formais (recomenda-se pelo menos duas) de condicionamento físico, nas quais sejam contempladas todas as etapas que compõem uma sessão padrão de exercício, com as etapas de aquecimento, parte principal e desaquecimento-relaxamento (volta à calma). Para que tal forma de reabilitação seja corretamente considerada, há necessidade de que protocolos de atendimento sejam usados. $\mathrm{O}$ atendimento deve ocorrer em serviços de atendimento ambulatorial, concebidos para o atendimento de pacientes encaminhados para a RCPM-NS, por meio de recursos humanos da área de treinamento físico (professores de educação física e/ou fisioterapeutas). O atendimento na RCPM-NS visa principalmente à adoção e manutenção da prática adequada de exercícios físicos.

\section{RESPONSABILIDADES}

MÉDICO ASSISTENTE: Deve encaminhar os pacientes com indicação de RCPM para os programas disponíveis. Deve se informar a respeito da RCPM e sempre que julgar necessário buscar a orientação do médico responsável pelo programa, visando à adoção de providências preliminares para que o paciente se apresente ao programa em condições ideais para o início das atividades. Providências principais: verificar se o quadro clínico do pacientes está estável (esquema medicamentoso otimizado), ter providenciado avaliação laboratorial recente, ter providenciado teste ergométrico em vigência de medicação de uso corrente.

MÉDICO RESPONSÁVEL: O médico responsável pelo programa deve estar adequadamente treinado em RCPM e ser conhecedor das técnicas de ressuscitação cardiopulmonar. Obrigações: a) manter treinada a equipe de reabilitação para eventual atendimento às emergências, inclusive no que diz respeito à parada cardiorrespiratória; b) avaliação pré-participação e liberação dos pacientes em condições de iniciar o programa; c) quando necessário, tomar as providências preliminares necessárias para que o paciente inicie a reabilitação em condições de usufruir os benefícios, em situação de maior segurança possível; d) elaborar a prescrição das atividades às quais o paciente vai ser submetido, em especial no que se refere ao exercício físico (zona limite para treinamento aeróbio, orientações para a execução de exercícios resistidos, interferência das drogas nas respostas fisiológicas, etc.). Portanto, ao médico que é o coordenador geral do programa de RCPM cumpre responder pela admissão com a obrigatória prescrição médica inicial e pela alta do paciente nas diversas fases da reabilitação (Conselho Federal de Medicina, Resolução 1236/87). Recomenda-se interação com o médico assistente do paciente, responsável pelo encaminhamento ao programa de RCPM, o qual sempre que necessário deve ser orientado.

MEMBROS COMPONENTES DA EQUIPE: Igualmente ao profissional médico, os outros membros da equipe (enfermeira, fisioterapeuta, professor de educação física, psicólogo, nutricionista, terapeuta ocupacional, assistente social) ao executarem suas respectivas funções devem seguir normas/regras que norteiam as atividades do programa. Cada profissional é responsável pelas atividades relacionadas às suas respectivas áreas de atuação.

GESTOR DO SISTEMA DE SAÚDE: Ao adotar política que implique priorizar ou questionar determinada modalidade terapêutica, inclusive a RCPM, deve estar devidamente fundamentado, considerando os estudos sobre custo-efetividade, o grau de recomendação e o nível de evidências científicas constantes em documentos de sociedades médicas (diretrizes/consensos/normas).

AUDITOR MÉDICO: Ao permitir ou negar determinado atendimento, deve estar fundamentado em estudos científicos. Para elaborar parecer sobre procedimentos, deve levar em consideração os estudos sobre custo-efetividade, o grau de recomendação e o nível de evidências científicas constantes em documentos de sociedades médicas (diretrizes/consensos/normas).

\section{BIBLIOGRAFIA}

1. Araújo CGS, Carvalho T, Castro CLB, Vivacqua RC, Moraes RS, Oliveira Filho J A. Normatização dos equipamentos e técnicas da reabilitação cardiovascular supervisionada. Arq Bras Cardiol 2004;83:448-52.

2. Moraes RS, Nóbrega ACL, Castro RRT, Negrão CE, Stein R, Serra SM, et al. Diretriz de reabilitação. Arq Bras Cardiol 2005.

3. Rehabilitation After Cardiovascular Diseases, with Special Emphasis on Developing Countries. Report of a WHO Expert Committee, 1993.

4. Goble A, Worcester M. Best practice guidelines for cardiac rehabilitation and secondary prevention 1999, Department of Human Services: Victoria, Australia.

5. Godoy M, et al. I Consenso Nacional de Reabilitação Cardiovascular. Arq Bras Cardiol 1997;69:267-91.

6. Ornish D, et al. Intensive life-style changes for reversal of coronary heart disease. J AMA 1998;16;280:2001-7. 
7. Niebauer J, Hambrecht R, Velich T, Hauer K, Marburger C, Kalberer B, et al. Attenuated progression of coronary artery disease after 6 years of multifactorial risk intervention: role of physical exercise. Circulation 1997;96:2534-41.

8. J olliffe J A, Rees K, Taylor RS, Thompson D, Oldridge N, Ebrahim S. Exercisebased rehabilitation for coronary heart disease [Cochrane Review]. Cochrane Database Syst Rev 2001;1:CD001800.

9. Ades PA, Pashkow FJ, Nestor J R. Cost-effectiveness of cardiac rehabilitation after myocardial infarction. J Cardiopulm Rehabil 1997;17:222-31.

10. Oldridge $\mathrm{N}$, et al. Number needed to treat in cardiac rehabilitation. J Cardiopulm Rehabil 2002;22:22-30.

11. Taylor RS, et al. Exercise-based rehabilitation for patients with coronary heart disease: systematic review and meta-analysis of randomized controlled trials. AmJ Med 2004; 15;116:682-92.

12. Hambrecht $\mathrm{R}$, et al. Percutaneous coronary angioplasty compared with exercise training in patients with stable coronary artery disease: a randomized trial. Circulation 2004;109:1371-8.

13. Belardinelli R, et al. Randomized, controlled trial of long-term moderate exercise training in chronic heart failure: effects on functional capacity, quality of life, and clinical outcome. Circulation 1999;99:1173-82.

14. Georgiou D. Cost-effectiveness analysis of long-term moderate exercise training in chronic heart failure. Am J Cardiol 2001;15;87:984-8.

15. Piepoli MF, Davos C, Francis DP, Coats AJ . ExTraM ATCH Collaborative. Exercise training meta-analysis of trials in patients with chronic heart failure (ExTraMATCH). BMJ 2004;328:189.

16. Whelton SP, Chin A, Xin X, He J . Effect of aerobic exercise on blood pressure: a meta-analysis of randomized, controlled trials. Ann Intern Med 2002;136:493-503.

17. Perkins J M, Collin J , Creasy TS, Fletcher EW, M orris PJ . Exercise training versus angioplasty for stable claudication: long and medium term results of a prospec tive, randomized trial. Eur J Vasc Endovasc Surg 1996;11:409-13.

18. Womack CJ , Ivey FM, Gardner AW, Macko RF. Fibrinolytic response to acute exercise in patients with peripheral arterial disease. Med Sci Sports Exerc 2000 33:214-9.

19. Hiatt WR, Regensteiner J G, Hargarten ME, Wolfel EE, Brass EP. Circulation. Benefit of exercise conditioning for patients with peripheral arterial disease. 1990; 81:602-9.

20. Verschuren WM, J acobs DR, Bloemberg DPM, et al. Serum total cholesterol and long-term coronary heart disease mortality in different cultures. Twenty-five-year follow-up of the Seven Coutries Study. J AMA 1995;274:131-6.

21. Ross R, J anssen I, Dawson J , Kungl AM, Kuk J L, Wong SL, et al. Exercise-induced reduction in obesity and insulin resistance in women: a randomized controlled trial. Obes Res 2004;12:789-98.

22. Watts K, Beye P, Siafarikas A, O'Driscoll G, J ones TW, Davis EA, et al. Effects of exercise training on vascular function in obese children. J Pediatr 2004;144: 620 5.

23. Miyatake N, Takanami S, Kawasaki Y, Fujii M. Relationship between visceral fat accumulation and physical fitness in J apanese women. Diabetes Res Clin Pract 2004;64:173-9.

24. Franks PW, Ekelund U, Brage S, Wong MY, Wareham NJ . Does the association of habitual physical activity with the metabolic syndrome differ by level of cardiorespiratory fitness? Diabetes Care 2004;27:1187-93.

25. Woo KS, Chook P, Yu CW, Sung RY, Qiao M, Leung SS, et al. Effects of diet and exercise on obesity-related vascular dysfunction in children. Circulation 2004 27;109:1981-6.

26. Anderson J W, Konz EC, Frederich RC, Wood CL. Long-term weight-loss maintenance: a meta-analysis of US studies. Am J Clin Nutr 2001;74:579-84.

27. Katzmarzyk PT, Church TS, Blair SN. Cardiorespiratory fitness attenuates the effects of the metabolic syndrome on all-cause and cardiovascular disease mortality in men. Arch Intern Med 2004;164:1092-97.

28. Herman WH, Hoerger TJ , Brandle M, Hicks K, Sorensen S, Zhang P, et al. Diabetes Prevention Program Research Group. The cost-effectiveness of life-style modification or metformin in preventing type 2 diabetes in adults with impaired glucose tolerance. Ann Intern Med 2005;1;142:323-32.

29. Salman GF, Mosier MC, Beasley BW, Calkins DR. Rehabilitation for patients with chronic obstructive pulmonary disease: meta-analysis of randomized controlled trials. J Gen Intern Med 2003;18:213-21.
30. Griffiths TL, Burr ML, Campbell IA, et al. Results at 1 year of outpatient multidisciplinary pulmonary rehabilitation: a randomized controlled trial. Lancet 2000; 355:362-8.

31. Goldstein RS, Gort EH, Guyatt GH, et al. Economic analysis of respiratory rehabilitation. Chest 1997;112:370-9.

32. Griffiths TL, Phillips CJ , Davies S, Burr ML, Campbell IA. Cost effectiveness of an outpatient multidisciplinary pulmonary rehabilitation programme. Thorax 2001 ; 56:779-84.

33. Golmohammadi K, J acobs P, Sin DD. Economic evaluation of a community-based pulmonary rehabilitation program for chronic obstructive pulmonary disease. Lung 2004;182:187-96.

34. Chapman RH, Stone PW, Sandberg EA, Bell C, Neumann PJ . A comprehensive league table of cost-utility ratios and a sub-table of " panel-worthy" studies. Med Decis Making 2000;20:451-67.

35. O'Brien BJ . Chapter 3. Cost effectiveness of ICD therapy: a review of published evidence. Can J Cardiol 2000;16:1307-12.

36. Wilson TM, Tanaka H. Meta-analysis of the age-associated decline in maximal aerobic capacity in men: relation to training status. Am J Physiol Heart Circ Physiol 2000;278:H829-34.

37. Garrett NA, Brasure M, Schmitz KH, Schultz MM, Huber MR. Physical inactivity: direct cost to a health plan. Am J Prev Med 2004;27:304-9.

38. Sakkas GK, Sargeant AJ , Mercer TH, Ball D, Koufaki P, Karatzaferi C, Naish PF. Changes in muscle morphology in dialysis patients after 6 months of aerobic exercise training. Nephrol Dial Transplant 2003;18:1854-61.

39. Miller BW, Cress CL, J ohnson ME, Nichols DH, Schnitzler MA. Exercise during hemodialysis decreases the use of antihypertensive medications. Am J Kidney Dis 2002;39:828-33.

40. Painter PL, Hector L, Ray K, Lynes L, Paul SM, Dodd M, et al. Effects of exercise training on coronary heart disease risk factors in renal transplant recipients. Am J f Kidney Dis 2003; 42:362-9.

41. Vaithilingam I, Polkinghorne KR, Atkins RC, Kerr PG. Time and exercise improve phosphate removal in hemodialysis patients. American J ournal of Kidney Diseases 2004;43:85-9.

42. Stack AG, Molony DA, Rives T, Tyson J , Murthy BVR. Association of Physica Activity with Mortality in the US Dialysis Population. AmJ Kidney Dis 2005;45:690701.

43. Vongvanich P, Paul-Labrador MJ , Merz CN. Safety of medically supervised exercise in a cardiac rehabilitation center. Am J Cardiol 1996;77:1383-5.

44. National Heart Foundation of New Zealand, Technical Report to Medical and Allied Professionals; Technical Report Number 78. 2002, National Heart Foundation: Auckland, NZ

45. U.S. Department of Health and Human Services, Cardiac Rehabilitation Clinica Practice Guideline Number 17. Public Health Service, Agency for Health Care Policy and Research, National Heart, Lung and Blood Institute 1995. 17(PN\#960672).

46. Benzer W, Oldridge NB. Current concepts in cardiac rehabilitation. J Clin Basic Cardiol 2001;4:211-9.

47. Freemantle $\mathrm{N}$, et al. Beta blockade after myocardial infarction: systematic review and meta regression analysis. BMJ 1999;318:1730-7.

48. Antiplatelet Trialists' Collaboration. Collaborative overview of randomized trials of antiplatelet therapy - Prevention of death, myocardial infarction, and stroke by prolonged antiplatelet therapy in various categories of patients. BMJ 1994; 308:81106.

49. Randomized trial of cholesterol lowering in 4444 patients with coronary heart disease: the Scandinavian Simvastatin Survival Study (4S). Lancet 1994;344:13839.

50. Kumana CR, et al. Gauging the impact of statins using number needed to treat. J AMA 1999;282:1899-901.

51. Long-term Intervention with Pravastatin in Ischaemic Disease (LIPID) Study Group. Prevention of cardiovascular events and death with pravastatin in patients with coronary heart disease and a broad range of initial cholesterol levels. N Engl J Med 1998;339:1349-57.

52. Grundy SM, Balady GJ, Criqui MH, Fletcher G, Greenland P, Hiratzka LF, et al Primary Prevention of Coronary Heart Disease: Guidance From Framingham: $A$ Statement for Healthcare Professionals From the AHA Task Force on Risk Reduction. Circulation 1998;97:1876-87. 